\title{
RSKManager - Um Jogo para Apoiar o Ensino de Gerência de Riscos em Projetos de Software
}

\author{
Lauriana Paludo \\ Mestrado em Computação Aplicada \\ Universidade do Vale do Itajaí - Univali \\ laurianapaludo@hotmail.com \\ André Luís Alice Raabe \\ Mestrado em Computação Aplicada \\ Programa de Pós Graduação em Educação \\ Universidade do Vale do Itajaí - Univali \\ raabe@univali.br \\ Fabiane Barreto Vavassori Benitti \\ Mestrado em Computação Aplicada \\ Universidade do Vale do Itajaí - Univali \\ fabiane.benitti@univali.br
}

\begin{abstract}
Resumo. O uso de jogos educacionais como complemento ao ensino pode promover a vivência de situações práticas do conhecimento abordado. Este artigo apresenta o jogo RSKManager desenvolvido para apoiar o ensino de Gerência de Riscos em Projetos de Software. O jogo simula o gerenciamento de um projeto de software envolvendo riscos de projeto, exercitando as atividades de identificação, análise, monitoramento e controle, planejamento e execução de respostas aos riscos do projeto. Foi elaborado um estudo sobre a efetividade do uso do jogo abordando tanto a capacidade de ensinar o conteúdo como a motivação de usá-lo como método de ensino. Os resultados demonstraram que o jogo tem potencial para auxiliar na aprendizagem de gerenciamento de riscos em projetos de software, contudo não houve comprovação estatística desses resultados nas avaliações realizadas.
\end{abstract}

Palavras-chaves. jogo educativo, gerenciamento de riscos em projetos de software, ensino de gerenciamento de projetos de software

Abstract. The use of educational games like complement to teaching can promote the experience of practical knowledge. This article presents the game RSKManager developed to support the teaching of Risk Management in Software Projects. The game simulates managing a software project involving risks project, exercising the activities of identification, analysis, monitoring and control, planning and execution of risk responses project. Was prepared a study on the effectiveness of using the game addressing both the ability to teach the content as motivation to use it as a teaching method. The results showed that the game has the potential to assist in learning risk management in software projects, but there was no statistical evidence of these results.

Keywords. education game, risks management in software projects, teaching of risks management in software projects.

\section{Introdução}


A área de conhecimento do Gerenciamento de Projetos de Software que lida com incertezas ou problemas mesmo antes que eles ocorram é a de Gerenciamento de Riscos. É uma área onde fatores que envolvem tecnologia, pessoas e processos podem gerar conflitos e influenciar de forma negativa e/ou positiva os objetivos de um projeto (PMI, 2008). Estes fatores podem ser tratados para minimizar seu impacto ou serem melhor aproveitados através do efetivo gerenciamento dos riscos do projeto.

O gerenciamento de riscos em projetos de software evoluiu nos últimos anos, passando de uma iniciativa pioneira (Boehm, 1988) para uma área de interesse atual, dada a diversidade de abordagens existentes, modelos e processos que lidam com o risco em um projeto (Menezes et al., 2013; Cooper, 2005) e trata fundamentalmente de se tomar as melhores decisões diante da ocorrência de uma situação desejada ou não.

A aquisição de competências e habilidades na tomada de decisões são aspectos do gerenciamento de projetos ligados à experiência e ao conhecimento adquirido através dessas experiências e que, particularmente, são difíceis de treinar em sala de aula (MINTZBERG apud CARBONE e GHOLSTON, 2004).

O uso de jogos na educação e especificamente na educação de engenharia de software tem sido discutido por vários autores tais como Navarro et al. (2004) e Taran (2007), que os tem recomendado usá-los como complemento às abordagens tradicionais de ensino em vez de um método sozinho. Utilizar abordagens de jogos e simulação permite ao estudante aprender fazendo, reduzindo assim a lacuna existente entre teoria e prática. Várias iniciativas apresentam jogos como complemento do ensino de gerência de projetos (PALUDO \& RAABE, 2010).

Nesse contexto, este artigo tem como objetivo apresentar o RSKManager, um jogo educativo para apoiar o ensino de gerenciamento de riscos em projetos de software. O desenvolvimento do jogo teve como base as definições de Alves (2010), que o caracterizam como jogo sério, ou seja, um jogo cujo foco é a aprendizagem, o treinamento para o desenvolvimento de habilidades específicas.

Para tal, o conteúdo do jogo é explorado através de estudos de caso contendo situações de incerteza que podem ocorrer em empresas de desenvolvimento de software e sob as quais o jogador deve tomar decisões que vão impactar nos objetivos do projeto.

O jogo exercita um processo para identificar, analisar, monitorar e responder aos riscos de um projeto de software, visando maximizar os resultados de eventos positivos e minimizar as conseqüências de eventos adversos. Seus objetivos educacionais são reconhecer e entender como é um processo de gerenciamento de riscos em projetos de software e entender e aplicar o gerenciamento dos riscos durante o desenvolvimento de um projeto de software.

Uma investigação do potencial pedagógico do RSKManager como ferramenta educacional de gerência de riscos em projetos de software foi realizada com três turmas de um curso de graduação em sistemas de informação tendo como primeira hipótese de pesquisa verificar se os estudantes que utilizam o jogo apresentavam melhora no aprendizado de gerenciamento de riscos em projetos de software em relação aos estudantes que não utilizaram o jogo. Uma segunda hipótese de pesquisa foi definida para verificar se os estudantes que utilizaram o jogo o consideravam uma ferramenta que motiva o aprendizado de gerenciamento de riscos em projetos de software.

O artigo está organizado pelas seguintes seções: primeira: introdução; segunda: visão geral da gerência de riscos em projetos de software; terceira: o ensino de gerenciamento de riscos em projetos de software; quarta: o jogo RSKManager; quinta: avaliações realizadas; sexta: conclusões e por último as referências.

\section{Gerência de Riscos em Projetos de Software}


Sob a ótica do gerenciamento de riscos em projetos, a definição de riscos do PMI (2008), aborda riscos de efeitos negativos e positivos, onde risco é um evento ou condição incerta que, se ocorrer, provocará um efeito positivo ou negativo sobre pelo menos um objetivo do projeto, como tempo, custo, escopo ou qualidade. Essa definição indica que as consequiências do risco não são, necessariamente, ruins ou negativas, os impactos sobre os resultados do projeto podem ser positivos ou negativos.

Groth e Hottell (2007), citam como exemplos de risco em projetos de software, a definição pobre de requisitos, a mudança de requisitos, o envolvimento insuficiente dos clientes, a falta de normas, a pobre ou inadequada metodologia, estimativas imprecisas, equipe insuficiente ou incorreta, tecnologia ultrapassada, moral baixa da equipe, problemas de qualidade, dependência de habilidades individuais, entre outros.

Associados a todo risco existem três fatores: probabilidade de que o risco ocorra; perda ou impacto gerado como consequência; e grau em que se pode mudar o resultado do risco.

Vários estudos têm sido feitos na área de identificação, análise e avaliação dos riscos, de uma maneira geral, sobre o processo de gerenciamento dos mesmos (PMI, 2008; SOFTEX, 2012; GUSMÃO e MOURA, 2005).

Para o PMI (2008), os objetivos do gerenciamento dos riscos são aumentar a probabilidade e o impacto dos eventos positivos e reduzir a probabilidade e o impacto dos eventos negativos no projeto. A gerência de riscos é a execução de atividades que viabilizam a identificação prévia e o tratamento de potenciais problemas. Essas atividades devem ser baseadas e centradas na comunicação e realizadas de forma cíclica e contínua dentro do processo de software com a finalidade de aumentar a qualidade do produto e do processo de desenvolvimento de software.

\section{O Ensino de Gerência de Riscos em Projetos de Software}

Em nível de graduação, nas Instituições de Ensino Superior (IES), o gerenciamento de riscos em projetos de software é ensinado com o conteúdo de gerenciamento de projetos. Este, por sua vez, é tratado nos currículos, ora como uma disciplina discreta, ora parte integrante da disciplina de Engenharia de Software e em alguns currículos, uma disciplina optativa.

Frequentemente, as disciplinas de Engenharia de Software e Gerência de Projetos são oferecidas entre o quarto e sétimo períodos, com carga horária de sessenta à cento e vinte horas. As ementas seguem os projetos pedagógicos de cada curso, são elaboradas pelas próprias IES e descrevem o conteúdo programático através de tópicos amplos, não detalhando de maneira específica o que realmente é abordado em cada área de conhecimento.

Os currículos de referência e diretrizes de ensino analisadas (ACM/IEEE-SE, 2004; CARBONE e GHOLSTON, 2004; SBC, 2005; CEEInf, 1999), recomendam que para desenvolver as competências para o gerenciamento de riscos em projetos de software, educação formal e práticas são essenciais e precisam ser consideradas nos currículos para preparar os estudantes para atuar como um gerente de projeto, contudo, essas mesmas recomendações tratam de forma muito vaga a distribuição de horas entre os conteúdos de gerenciamento de projetos.

Consequentemente, a carga horária destinada ao gerenciamento de riscos, em função dos demais conteúdos, tende a ser pequena para ensinar e exercitar o conhecimento ao ponto de atingir níveis cognitivos mais altos com ênfase na transferência de experiências para situações do mundo real. Em geral, os conteúdos tem como base os conceitos apresentadas no PMBOK.

Pode-se dizer então, que o ensino de gerenciamento de riscos em projetos de software fica condicionado a fatores como tempo e conhecimento do professor, não sendo um conteúdo recomendado como referência de ensino ou mesmo obrigatório nas disciplinas e pode não receber espaço suficiente para se exercitar conhecimentos teóricos e práticos que levem o estudante a aplicar na prática o conhecimento adquirido. 


\section{O Jogo RSKManager}

O jogo RSKManager foi concebido com a intenção de proporcionar um ambiente diferente de aprendizagem que permitisse aos estudantes reforçar o aprendizado de gerenciamento de riscos em projetos de software. Aborda a identificação, a análise, o planejamento de respostas, o monitoramento e a execução de ações de tratamento de riscos em projetos de software. No jogo, os riscos são considerados como positivos ou negativos em relação aos objetivos de custo e prazo de um projeto de software.

Foi projetado para ser utilizado de forma individual e como complemento às aulas tradicionais ou de cursos à distância para o público alvo de estudantes de graduação em cursos de Computação e/ou profissionais da área com conhecimento básico de Engenharia de Software e Gerenciamento de Projeto ou que desejam iniciar seus estudos em Gerenciamento de Riscos em Projetos de Software.

Os problemas que o jogador deve resolver são apresentados por estudos de caso de projetos de software que podem ser configurados pelo professor da disciplina a partir de experiências, contextos relevantes da realidade e nível de complexidade que deseja abordar, customizando, entre outros, restrições de prazo, custo, riscos, análise e respostas adequadas aos riscos. Esses parâmetros são utilizados para o feedback dado ao aluno de seu desempenho no jogo. Vence o jogo, o jogador que finalizar o projeto dentro dos critérios de aceitação planejados no estudo de caso, simulando a entrega do projeto para o cliente.

A Figura 1 apresenta a interface do jogo de ambiente de desenvolvimento chamada quando se inicia um jogo. Nela são apresentadas as atividades correspondentes a fase do desenvolvimento à esquerda, na área central o processo de gerenciamento de riscos com os botões de identificar risco, analisar e monitorar risco, executar ação para tratamento de risco e excluir risco, nessa ordem, e à direita da tela a visualização da situação do projeto em relação ao prazo e custo decorridos.

O status do risco, na área central de gerenciamento de riscos, pode ser visualizado pela imagem representativa de um triângulo amarelo para riscos identificados, de ponto de interrogação para risco não identificado, de uma bomba se o risco for negativo ou de uma estrela se o risco for positivo.

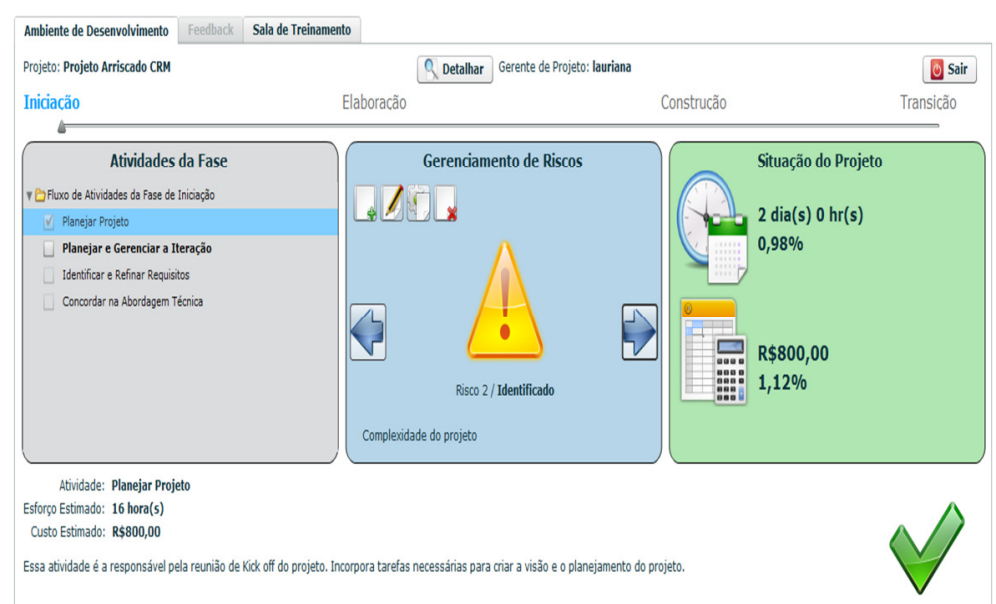

Figura 1 - Interface de Ambiente de Desenvolvimento do RSKManager. 
Os jogadores iniciam o jogo identificando os riscos do projeto selecionado. A identificação cria a lista de riscos do projeto e a partir dos riscos identificados o jogador pode realizar as atividades de análise, monitoramento e tratamento dos riscos.

Para identificar riscos, o jogador deve selecionar, de um checklist de riscos, apenas os riscos que pertencem ao cenário do projeto de software escolhido. A análise dos riscos baseia-se na seleção de uma categoria para o risco, na definição se o risco é um evento positivo ou negativo, de uma probabilidade - alta, média ou baixa - de um impacto - alto, médio ou baixo - e uma prioridade - alta, moderada ou baixa - para os riscos identificados. Planejar respostas aos riscos consiste na seleção de uma estratégia de ação - mitigar, melhorar, aceitar ou transferir - e de ações específicas para o tratamento dos riscos. Para cada ação estratégica planejada, a fase do desenvolvimento em que ela deve ser executada para garantir que tenha efeito no projeto deve ser também definida. Monitorar riscos envolve o acompanhamento dos riscos identificados, a identificação de novos riscos e a aplicação do tratamento planejado aos riscos.

$\mathrm{O}$ avanço no jogo acontece com a realização das atividades do processo de software, simulando o desenvolvimento de um projeto. Como o processo de gerenciamento de riscos é cíclico, ele deve ser realizado em todas as fases do desenvolvimento. Uma vez executada a fase de iniciação, o jogo pode ser finalizado a qualquer momento caso os riscos não forem corretamente gerenciados.

As escolhas do jogador vão formando seu feedback de desempenho e afetam diretamente os resultados do projeto em termos de custo e prazo, ou seja, erros de identificação, análise, priorização, estratégia e tratamento para riscos negativos ou positivos recebem um percentual de acréscimo no custo e no prazo do projeto em relação ao total estimado para o projeto.

O feedback do jogador pode ser visualizado através de um feedback parcial por fase do desenvolvimento - para cada risco ocorrido é apresentada uma visualização indicando se as escolhas do jogador estão certas ou erradas - e de um feedback geral, onde os acertos e erros que o jogador fez durante o jogo são visualizados através de gráficos de pizza. As respostas do jogador são visualmente organizadas por atividades do gerenciamento de riscos em identificação, análise, priorização, estratégia e tratamento dado ao risco. A Figura 2 apresenta a interface de feedback parcial e geral do jogador.

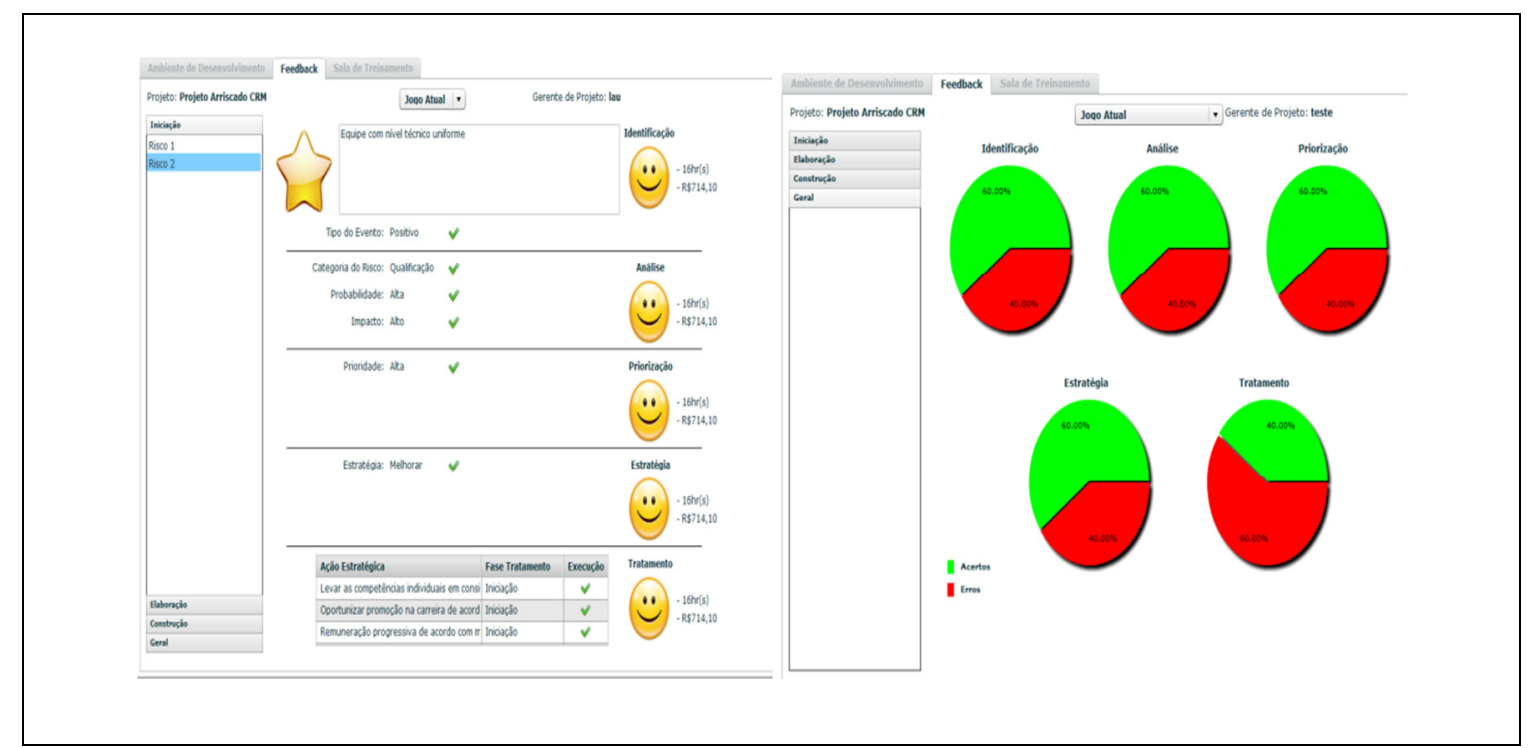

Figura 2 - Interface de Feedback parcial e geral do jogador.

O feedback é uma comparação da resposta definida como certa pelo professor no estudo de caso e da resposta dada pelo jogador. Como essa opção de certo ou errado é muito subjetiva, para cada projeto é definido na elaboração do estudo de caso um limite de desvio percentual 
máximo relativo ao tempo e ao custo total estimados para o projeto que serve também para encerrar o jogo quando esse limite for atingido. Quando o jogo for finalizado ou chegar ao seu fim, um desempenho final em relação aos prazos e custos configurados e realizados pelo jogador é apresentado.

O jogador pode sair do jogo e continuá-lo mais tarde que o progresso do jogo será salvo automaticamente. Pode também acessar a sala de treinamento que tem como objetivo a realização de estudos, consulta a materiais complementares de gerenciamento de riscos em projetos de software e informações sobre o próprio jogo. Simulando um processo de treinamento real, cada acesso a sala de treinamento gera um esforço e um custo para o projeto.

Os jogos de cada usuário são armazenados numa pasta users, no diretório de instalação do jogo. O usuário pode consultar seu jogo atual e outros jogos que jogou para acompanhar sua evolução no gerenciamento de riscos, também pode enviar essa pasta ao professor para que ele possa avaliá-lo e intervir mais pontualmente em seu aprendizado.

O professor pode acessar através de um login de administrador, uma interface onde pode criptografar/descriptografar os estudos de caso e selecionar estudos de caso para análise ou avaliação. As opções de criptografar/descriptografar garantem que o aluno não modifique os estudos de caso.

\section{Avaliação do uso do jogo}

Foram realizadas três avaliações do uso do jogo com alunos de um curso superior de Bacharelado em Sistemas de Informação com o objetivo de analisar a efetividade do jogo como ferramenta de apoio ao ensino de gerenciamento de riscos em projetos de software. Uma, dessas três avaliações, foi realizada com o design de grupo de controle. Os instrumentos de pesquisa utilizados envolveram um questionário para identificar o perfil dos alunos, um pré-teste, uso do jogo, pós-teste e uma avaliação qualitativa da percepção dos alunos sobre o jogo.

Os alunos convidados a participar das avaliações estavam regularmente matriculados ou já tinham cursado as disciplinas de Engenharia de Software e Gerência de Projetos de Software caracterizando o público alvo recomendado para o uso do jogo.

A primeira e a segunda avaliação iniciaram com a assinatura do termo de consentimento em participar da pesquisa, seguida de uma apresentação das regras do jogo pela pesquisadora. Na sequencia, um questionário de perfil do participante foi preenchido, onde cada um avaliou seu conhecimento, experiência, habilidade e interesse em gerenciamento de riscos em projetos de software a partir de uma escala variando de zero (nenhum) a cinco (excelente).

Foi aplicado um pré-teste contendo doze questões de conhecimentos em gerenciamento de riscos em projetos de software com o objetivo de obter um parâmetro de comparação ao fim do uso do jogo com segunda aplicação do questionário (pós-teste) e verificar se houve progresso no aprendizado, avaliando o conhecimento de gerência de riscos dos participantes nos conceitos abordados pelo jogo. As questões de pré e pós-teste foram de múltipla escolha, sendo apenas uma resposta correta entre quatro opções de respostas e envolveram definições e exemplos de riscos de projeto, atividades do gerenciamento de riscos e estratégias de tratamento de riscos. Pré e pós-teste foram avaliados através da contagem de respostas corretas e comparação de quantidades. Ambos os testes foram respondidos individualmente e sem consulta.

Ao fim do pré-teste, o jogo RSKManager foi jogado uma única vez até o seu final. Os participantes responderam ao pós-teste e na sequencia a um questionário de avaliação do uso do jogo. A Tabela 1 apresenta os resultados do teste de hipóteses da primeira avaliação realizada com 13 alunos e da segunda avaliação com 05 alunos.

Tabela 1 - Resultados do teste de hipóteses da primeira avaliação com alunos. 


\begin{tabular}{|r|c|c|}
\hline \multicolumn{3}{|c|}{ Teste de Hipóteses } \\
\hline & Avaliação 1 & Avaliação 2 \\
\hline $\boldsymbol{\alpha}$ & 0,05 & 0,05 \\
\hline gl & 12 & 4 \\
\hline t observado & 4,935 & 3,060 \\
\hline p-value & 0,000167958 & 0,004290459 \\
\hline
\end{tabular}

Na primeira avaliação, o teste $t$ de student apresentou o valor t calculado de 4,935 e o valor de $\mathrm{t}$ crítico para esta amostra de 1,78 demonstrando que esse resultado ( $\mathrm{t}$ calculado $>\mathrm{t}$ crítico) significa que o uso do jogo apresentou indícios de produzir um efeito positivo no aprendizado de gerenciamento de riscos em projetos de software. Pode-se dizer que a média de acertos após o jogo, de 8,08 foi significativamente melhor $(\alpha=0,05)$ do que antes do jogo de 6,23 .

Para a segunda avaliação, o teste t de student apresentou o valor t calculado de 3,060 e o valor de $\mathrm{t}$ crítico para esta amostra de 2,132 demonstrando que esse resultado ( $\mathrm{t}$ calculado $>\mathrm{t}$ crítico) significa que o uso do jogo parece ter produzido um efeito positivo no aprendizado de gerenciamento de riscos em projetos de software. Pode-se dizer que a média de acertos após o jogo, de 8,8 foi significativamente melhor $(\alpha=0,05)$ do que antes do jogo de 7 .

A Tabela 2 apresenta os valores do teste $t$ calculado e t crítico por nível de aprendizagem dos participantes das duas avaliações.

Tabela 2 - Análise quantitativa por nível de aprendizagem das duas avaliações.

\begin{tabular}{|l|c|c|c|c|c|c|}
\cline { 2 - 7 } \multicolumn{1}{c|}{} & \multicolumn{3}{c|}{ Avaliação 1 } & \multicolumn{3}{c|}{ Avaliação 2 } \\
\cline { 2 - 7 } \multicolumn{1}{c|}{} & Lembrança & Compreensão & Aplicação & Lembrança & Compreensão & Aplicação \\
\hline t calculado & 3,06 & 1,89 & 1,43 & 2,23 & 0,46 & 0,66 \\
\hline t crítico & 1,78 & 1,78 & 1,78 & 2,13 & 2,13 & 2,13 \\
\hline
\end{tabular}

Verificou-se, com base nos valores da Tabela 2, que o uso do jogo, na primeira avaliação, demonstrou indícios de produzir um efeito positivo no aprendizado de gerenciamento de riscos em projetos nos níveis de Lembrança e Compreensão da taxonomia, podendo-se dizer que a média de acertos após o uso do jogo para esses níveis foram significativamente melhores $(\alpha=$ $0,05)$ do que antes do jogo. Já para o nível de Aplicação, o valor do t calculado é menor que o valor do t crítico, não aparecendo indícios nesse experimento de que os estudantes aplicam melhor o gerenciamento de riscos em projetos de software após o uso do jogo.

Na segunda avaliação, conforme apresentado na Tabela 2 acima, verificou-se que o uso do jogo demonstrou indícios de produzir um efeito positivo no aprendizado de gerenciamento de riscos em projetos somente no nível de Lembrança da taxonomia, podendo-se dizer que a média de acertos após o uso do jogo para esse nível foi significativamente melhor $(\alpha=0,05)$ do que antes do jogo. Já para os níveis de Compreensão e Aplicação, o valor do t calculado é menor que o valor do $\mathrm{t}$ crítico, não aparecendo indícios nesse experimento de que os estudantes compreendem ou aplicam melhor o gerenciamento de riscos em projetos de software após o uso do jogo.

\subsection{Terceira Avaliação do Jogo}

$\mathrm{Na}$ terceira avaliação do uso do jogo, os participantes foram divididos de maneira aleatória. em dois grupos (experimental e controle), sendo estes identificados, respectivamente, por grupo A (10 alunos) e grupo B (07 alunos).

A avaliação utilizou os mesmos instrumentos de pesquisa das avaliações anteriores com exceção do questionário de avaliação qualitativa que foi substituído por uma entrevista semi- 
estruturada, baseada nas perguntas do questionário, com dois alunos voluntários que jogaram o jogo. O questionário foi substituído por ter demonstrado ser cansativo de responder e muito extenso, principalmente por ser aplicado após o uso do jogo.

Analisando as médias dos 17 alunos que participaram da avaliação tanto no pré-teste quanto no pós-teste, apresentadas na Tabela 3, a média da turma no pré-teste foi de 4,05 e no pós-teste de 5,23 com um desvio padrão no pré-teste de 1,08 e no pós-teste de 1,30, sugerindo que de modo geral os participantes aumentaram a média de acertos no pós-teste.

Tabela 3 - Resultados estatísticos da terceira avaliação com alunos.

\begin{tabular}{|l|c|c|c|c|c|c|}
\cline { 2 - 7 } \multicolumn{1}{c|}{} & \multicolumn{3}{c|}{ Pré-teste } & \multicolumn{3}{c|}{ Pós-teste } \\
\cline { 2 - 7 } \multicolumn{1}{c|}{} & Participantes & GE & GC & Participantes & GE & GC \\
\hline Média acertos & 4,05 & 3,9 & 4,28 & 5,23 & 5,4 & 5,0 \\
\hline Desvio Padrão & 1,08 & 1,37 & 0,48 & 1,30 & 1,42 & 1,15 \\
\hline
\end{tabular}

Considerando apenas os sete alunos que participaram do grupo de controle, a média deste grupo no pré-teste foi de 4,28, enquanto no pós-teste a média do grupo foi de 5,0. Isso mostra que os alunos do grupo de controle tiveram um desempenho melhor no segundo teste. Contudo, o desvio padrão das notas do grupo de controle variou muito do pré $(0,48)$ e pós-teste $(1,15)$ indicando que o grupo obteve uma variação em torno da média alta e as médias de pré e pósteste muito próximas, não sendo possível afirmar que são significativamente diferentes e dificultando uma análise mais completa. Sugere-se a aplicação de uma nova avaliação com um grupo mais homogêneo para que a variação dentro do mesmo grupo não seja tão diferente.

A Tabela 4 apresenta os valores do teste t calculado e crítico por nível de aprendizagem dos participantes do grupo experimental da terceira avaliação.

Tabela 4 - Análise quantitativa por nível de aprendizagem da terceira avaliação.

\begin{tabular}{|l|c|c|c|}
\cline { 2 - 4 } \multicolumn{1}{c|}{} & Lembrança & Compreensão & Aplicação \\
\hline $\mathrm{t}$ calculado & 1,15 & 1,46 & 0,90 \\
\hline $\mathrm{t}$ crítico & 1,83 & 1,83 & 1,83 \\
\hline
\end{tabular}

Observou-se que os valores de $\mathrm{t}$ calculados são menores que os valores do $\mathrm{t}$ crítico para os três níveis da taxonomia analisados, podendo-se dizer que a média de acertos após o uso do jogo para esse grupo não foi significativamente melhor $(\alpha=0,05)$ do que antes do jogo. Isso quer dizer que para esse grupo não foi encontrado indícios de que o uso do jogo produziu um efeito positivo nos níveis de lembrança, compreensão e/ou aplicação do gerenciamento de riscos em projetos de software.

\section{Conclusões}

Analisando o resultado geral das avaliações realizadas é possível afirmar que o jogo RSKManager tem potencial para auxiliar na aprendizagem de gerenciamento de riscos de projetos de software, principalmente em nível de lembrança do conhecimento abordado. Contudo, não foi possível comprovar estatisticamente que os resultados da terceira avaliação são significativos, rejeitando a hipótese de pesquisa de que os estudantes que utilizam o jogo apresentam melhora no aprendizado de gerenciamento de riscos em projetos de software em relação aos estudantes que não utilizam o jogo.

Em relação à percepção dos participantes sobre o jogo, nas três avaliações, os alunos que utilizaram o jogo, o julgaram uma ferramenta que motiva o aprendizado em gerência de riscos 
em projetos de software. Como pontos fortes foram apontados: permitir sair e continuar o jogo, o layout da interface, facilidade de utilização e entendimento, o ambiente amigável, a abrangência do processo de gerenciamento de riscos que o jogo aborda, relevância do conteúdo, aproximação com situações do mundo real.

Como sugestões de melhoria e continuidade deste trabalho, em relação ao aspecto experiência de usuário, a imersão, desafio e ritmo, explorar mais a interatividade do ambiente, animações e recursos de som, diminuir textos, aumentar a interação entre os usuários com um ranking, colaboração, multiplayer; flexibilizar o feedback dado ao jogador, criar níveis de dificuldade, ampliar o uso de técnicas para identificação de riscos, transitar da análise qualitativa dos riscos para a quantitativa, criar uma interface para o cadastro de estudos de caso.

Em relação às ameaças a validade, para a ameaça de construção da avaliação e o fato dos alunos convidados não estarem presentes no momento da aplicação, os alunos foram previamente avisados e consultados quanto à data de aplicação da avaliação e a mesma foi utilizada pelo professor na pontuação do aluno na disciplina.

Quanto à ameaça interna da validade dos resultados, de que os alunos pudessem estudar entre o pré e o pós-teste, as atividades da avaliação foram realizadas todas em sequencia e sem intervalo.

Para a ameaça interna de incompatibilidade entre pré e pós-teste, foi adotado o procedimento em que os dois instrumentos teriam as mesmas questões ordenadas de maneira diferente. Ao responderem o pós-teste, os alunos foram informados que os instrumentos eram diferentes e que analisassem com calma as respostas. Testes diferentes não foram utilizados em função da ameaça de incompatibilidade entre os instrumentos, ou seja, de um deles ser mais complexo ou mais simples que o outro levando a um falso resultado.

Como ameaça externa à avaliação, foi levantada a possibilidade do participante ter conhecimento ou experiência prévia em gerenciamento de riscos em níveis avançados. Com base nos resultados do questionário de perfil do participante e do pré e pós-teste, pode-se verificar que nenhum dos participantes apresentou conhecimento ou experiência que pudesse descaracterizar os resultados da avaliação, não sendo necessário a eliminação de nenhum participante de nenhuma avaliação.

Em relação a ameaça de conclusão identificada, referindo-se ao comprometimento da pesquisadora com a pesquisa e o resultado obtido, todos os passos da pesquisa foram documentados e podem ser replicados por outros pesquisadores, permitindo ainda outras interpretações, novas pesquisas e mesmo a comparação de resultados obtidos em pesquisas.

Admite-se, como limitação deste trabalho, que o monitoramento de riscos que deve ocorrer ao longo do desenvolvimento de um projeto de software não contempla toda a evolução da existência do risco. A implementação da modificação dos parâmetros da análise em uma próxima versão do jogo pode auxiliar a resolver esse problema.

\section{Referências}

ACM/IEEE-SE. Task Force on Computing Curricula (2004) "Software Engineering 2004 Curriculum Guidelines for Undergraduate Degree Programs in Software Engineering ", http://sites.computer.org/ccse/SE2004Volume.pdf, um volume da série Computing Curricula Series, August 23, 2004.

ALVES, E. M. S. P. Arquitetura Pedagógica Aplicada ao Ensino de Gerenciamento de Projetos: a percepção do professor e dos alunos acerca da incorporação de um artefato tecnológico no planejamento didático. Mestrado Acadêmico em Computação Aplicada. UNIVALI, Itajaí, SC, 2010. 
BOEHM, Barry. A spiral model of software development and enhancement. IEEE Computer, v. 21, n. 5, p. 61-72, 1988.

CARBOnE, Thomas A.; GHOLSTON, Sampsom. Project Manager Skill Development: A Survey of Programs and Practitioners. Engineering Management Journal Vol. 16 No. 3 September, 2004.

CEEInf. Diretrizes Curriculares de Cursos da Área de Computação e Informática. Comissão de Especialistas de Ensino de Computação d Informática - CEEInf. Secretaria de Educação Superior do MEC (SESu/MEC). 1999. Disponível em www.inf.ufrgs.br/sitelengcomp/docs/diretriz.pdf. Acessado em novembro de 2008.

COOPER, Dale F. et al. Project risk management guidelines: managing risk in large projects and complex procurements. Chichester, UK: John Wiley and Sons, 2005.

GUSMAO, Cristine Martins Gomes de. MOURA, Hermano Perrelli de. Gerência de Risco em Processos de Qualidade de Software: uma Análise Comparativa. IV Simpósio Brasileiro de Qualidade de Software - SBQS 2005. Porto Alegre - RS, Brasil.

GROTH, Dennis P. and HOTTELL, Matthew P. How Students Perceive Risk: A Study of Senior Capstone Project Teams. Software Engineering Education e Training, 2007.

MENEZES JR, J.; GUSMÃO, C.; MOURA, H. Defining Indicators for Risk Assessment in Software Development Projects. CLEI ELECTRONIC JOURNAL, Volume 16, Número 1, Artigo 10, Abril, 2013.

NAVARRO, E.; BAKER, A.; HOEK. SIMSE: An Interactive Simulation Game For Software Engineering Education. School of Information and Computer Science University of California Irvine, 2004.

PALUDO, L. RAABE, A. L. A. Análise de Jogos Educativos de Computador para Gerência de Projetos de Software. XVIII Workshop sobre Educação em Computação (WEI), Belo Horizonte, Minas Gerais, 2010.

PMI - Project Management Institute, "A Guide to the Project Management Body of Knowledge". 4th edition, Project Management Institute (PMI), Newtown Square, Pennsylvania, USA, 2008.

SBC. CR2005 - Currículo de Referência da SBC para Cursos de Graduação em Bacharelado em Ciência da Computação e Engenharia de Computação. Proposta versão 2005. Sociedade Brasileira de Computação (SBC). Disponível em www.sbc.org.br. Acessado em outubro de 2008.

SOFTEX, MPS . BR - Guia Geral MPS de Software:2012. Associação para Promoção da Excelência do Software Brasileiro, 2012.

TARAN, Gil. Using Games in Software Engineering Education to Teach Risk Management. 20th Conference on Software Engineering Education \& Training (CSEET'07), 2007. 